Disease, Metabolism and Reproduction in the Toxic Response to Drugs and Other Chemicals Arch. Toxicol., Suppl. 7, 194-207 (1984)

(C) by Springer-Verlag 1984

\title{
Structural Characteristics of Compounds That can be Activated to Chemically Reactive Metabolites: Use for a Prediction of a Carcinogenic Potential
}

\author{
W. K. Lutz \\ Institute of Toxicology, ETH and University of Zurich, 8603 Schwerzenbach, Switzerland
}

\begin{abstract}
Many mutagens and carcinogens act via covalent interaction of metabolic intermediates with DNA in the target cell. This report groups those structural elements which are often found to form the basis for a metabolism to such chemically reactive metabolites. Compounds which are chemically reactive per se and which do not require metabolic activation form group 1. Group 2 comprises of olefins and aromatic hydrocarbons where the oxidation via an epoxide can be responsible for the generation of reactive species. Aromatic amines, hydrazines, and nitrosamines form group 3 requiring an oxidation of a nitrogen atom or of a carbon atom in alpha position to a nitrosated amine. Group 4 compounds are halogenated hydrocarbons which can either give rise to radicals or can form an-olefin (group 2) upon dehydrohalogenation. Group 5 compounds depend upon some preceding enzymatic activity either not available in the target cell or acting on positions in the molecule which are not directly involved in the subsequent formation of electrophilic atoms. Examples for each group are taken from the "List of Chemicals and Industrial Processes Associated with Cancer in Humans" as compiled by the International Agency for the Research on Cancer, and it is shown that $91 \%$ of the organic carcinogens would have been detected on the basis of structural elements characteristic for group 1-5. As opposed to this very high sensitivity, the specificity (the true negative fraction) of using this approach as a short-term test for carcinogenicity is shown to be bad because detoxification pathways have so far not been taken into account. These competing processes are so complex, however, that either only very extensive knowledge about pharmacokinetics, stability, and reactivity will be required or that in vivo systems have to be used to predict, on a quantitative basis, the damage expected on the DNA. DNA-binding experiments in vivo are presented with benzene and toluene to
\end{abstract}

Abbreviations. CBI, Covalent Binding Index $=$ (umol substance bound per mol DNA nucleotides)/(mmol substance administered per $\mathrm{kg}$ body weight); HCH, hexachlorocyclohexane 
demonstrate one possible way for an experimental assessment and it is shown that the detoxification reaction at the methyl group available only in toluene gives rise to a reduction by at least a factor of forty for the binding to rat liver DNA. This quantitative approach available with DNA-binding tests in vivo, also allows evaluation as to whether reactive metabolites and their DNA binding are always the most important single activities contributing to the overall carcinogenicity of a chemical. With the example of the livertumor inducing hexachlorocyclohexane isomers it is shown that situations will be found where reactive metabolites are formed and DNA binding in vivo is measurable but where this activity cannot be the decisive mode of carcinogenic action. It is concluded that the lack of structural elements known to become potentially reactive does not guarantee the lack of a carcinogenic potential.

Key words: Structure-activity relationship - Reactive intermediates Metabolic activation - DNA Binding - Covalent binding index Carcinogens - Benzene - Toluene - Hexachlorocyclohexane - Lindane

It has always been a dream of chemists to predict, on the basis of structure, the activity of chemicals in living systems. One is not aware though of any example where this approach would not have ended at some point with an exception to a rule so carefully established.

In the last decade, a new wave of optimism swept over the biologically interested chemists after the exciting finding that many well-known strong carcinogens belonging to a number of different chemical classes were shown to be metabolized via electrophilic, i.e., chemically reactive intermediates (Miller 1970) and that their covalent binding to DNA seemed to be an important early event in the chemical induction of a tumor (Lutz 1979). No wonder that many chemists tried to find ways to predict the carcinogenicity on the basis of critical structural elements. The most elaborate try came from Cramer et al. (1978) who developed a decision tree system where organic chemicals could be placed into one of three classes I, II, or III, on the basis of 33 questions. These classes were meant to reflect a presumption of low, moderate, or serious toxicity, and the authors claimed that so far the procedure had not resulted in any underestimation of toxicity. The analysis reported by Ashby (1978) and by Oesch (1982) revealed a much more complicated situation as soon as quantitative assessments are attempted.

The following report will try to answer three questions. How sensitive is a short-term test for carcinogenicity based exclusively on critical structural elements? How good is the specificity for this type of test, i.e., how large is the fraction of non-carcinogens lacking a critical structural element? If a reactive metabolite can be formed, is this activity always the most important for its over-all carcinogenic activity? 


\section{Question 1: How Sensitive is a Test Based on Critical Structural Elements?}

In order to answer the question of sensitivity, there must be some base list of chemicals that have been shown to be carcinogenic. In this case, rather arbitrarily, the "List of Chemicals and Industrial Processes Associated with Cancer in Humans" as compiled by the International Agency for the Research on Cancer (IARC 1980) was chosen. It has to be mentioned that the evidence for carcinogenicity is admittedly inadequate for a number of the 54 compounds listed there, but the list still seemed appropriate for an evaluation of critical structural elements.

A comparison of the chemical structures revealed five groups where a formation of reactive intermediates has to be envisaged. These will be presented in the following sections.

\section{Group 1: Chemically Reactive per se}

Figure 1 shows a number of structural elements known to the chemist to be electrophilically reactive. The list comprises of the strained three-membered rings containing oxygen ( $a$, epoxides), nitrogen ( $b$, aziridines), and sulfur ( $c$, epithio derivatives); halogenated alkanes ( $\mathrm{d}$ and $\mathrm{e}$ ) where the chloride becomes a favourable leaving group in a nucleophilic attack because of neighbouring effects by nitrogen (d, mustards) or oxygen (e, ethers): some alkylating and

Table 1. Group 1 of IARC 1980 list of carcinogens: chemically, reactive per se (13 of 54)

\begin{tabular}{ll}
\hline a) Epoxides & 27. Epichlorohydrin \\
& 28. Ethylene oxide \\
& 23. Dieldrin (also group 4) \\
b) Aziridines & 52. Triaziquone \\
& 53. Thiotepa \\
c) Epithio derivatives & $*$ Epithiobutyronitrile \\
d) 2-Chloroethyl derivatives & 12. Chlornaphazine \\
& 16. Chlorambucil \\
& 21. Cyclophosphamide (also group 5) \\
& 37. Melphalan \\
e) Chloromethyl derivatives & 38. Mustard gas \\
f) Sulfates, sulfonates & 13. Bis(chloromethyl) ether \\
g) Acid halides & 26. Dimethyl sulfate \\
h) Aldehydes & 25. Dimethyl carbamoylchloride \\
i/j) N-Nitroso derivatives & $*$ Formaldehyde \\
& $*$ Methyinitrosourea \\
\hline
\end{tabular}

Numbering according to IARC (1980)

* Additional carcinogens 
acylating agents ( $\mathrm{f}$ and $\mathrm{g}$ ); formaldehyde as most reactive representative of the class of carbonyl compounds and potential crosslinking agent (h); and two derivatives of the important class of N-nitroso derivatives, some of which, like ureas and primary amines are known to undergo spontaneous decay to alkylating agents. A reaction with a primary amine is depicted in Fig. 1, as one possible nucleophile present in protein and nucleie acids.

Although group 1 chemicals do not require metabolic activation to become reactive, this group has been included here and even put first because 13 out of the 54 IARC carcinogens belong to this class (Table 1). About half of these are cytostatic agents where a reactivity towards DNA is a prerequisite for its therapeutic effectiveness. Aldehydes and N-nitroso derivatives have been added because of their wide occurrence.

a)
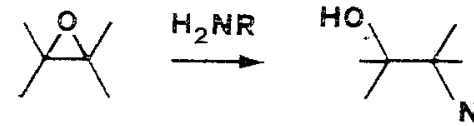

NHR

b)

$R-N J$

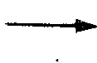

$\mathrm{R}^{1} \mathrm{NHCH}_{2} \mathrm{CH}_{2} \mathrm{NHR}$ c)
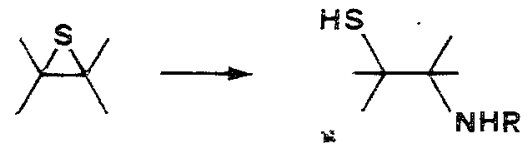

d) $\mathrm{HCH}_{2} \mathrm{CH}_{2} \mathrm{Cl} \rightarrow \mathrm{NCH}_{2} \mathrm{CH}_{2} \mathrm{NHR}$ e)

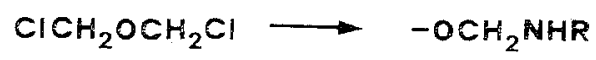

f)<smiles>C[Sb](C)(=O)=O</smiles><smiles>[R]NC(C)[I-]C</smiles>

9)<smiles>CN(C)C(=O)Cl</smiles>

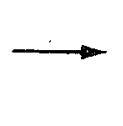<smiles>[R]NC(=O)N(C)C</smiles>

h)<smiles>[R]NC(N[R])NC([R])C(=O)O</smiles>

i)

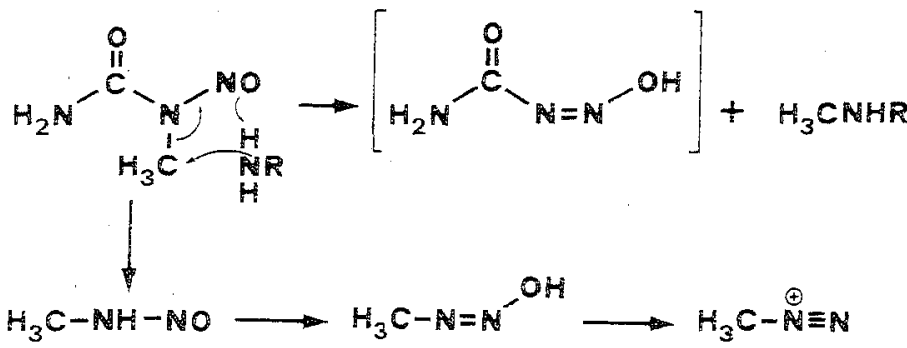

Fig. $1 \mathrm{a}-\mathbf{j}$. Structures of chemically reactive carcinogens selected from the IARC (1980) list of carcinogens. A primary amine $\mathrm{H}_{2} \mathrm{NR}$ is shown as the nucleophilic reaction partner. a Epoxides, b aziridines, c epithio derivatives, d 2-chloroethyl derivatives (mustards), e chloromethylderivatives, f sulfates, sulfonates, $\mathbf{g}$ acid halides (dimethyl carbamoylchloride), h aldehydes (formaldehyde), $\mathrm{i} / \mathrm{j} \mathrm{N}$-nitroso-derivatives of a urea and of a primary amine; $\rightarrow j$ hydrolysis of the labilized amide bond 


\section{Group 2: Substituted Olefins}

A large group is formed by those compounds which contain a carbon-carbon double bond (Fig. 2), including aromatic systems such as those found in polynuclear aromatic hydrocarbons. It is interesting to note that all the respective representatives taken from the IARC 1980 list of carcinogens (Table 2) have their double bond conjugated (c, d) or unsymmetrically substituted with electrophilic atoms (a, b). The reactive metabolite derived therefrom is an epoxide formed by the monooxygenase system which is discussed in more detail in subsequent presentations. Among the standard list of 54, 13 compounds would have been perceived as potential carcinogens (Table 2).

\section{Group 3: Oxidation of Nitrogen or of an alpha-Carbon of a Nitrosamine}

Aromatic amines have been responsible for the high incidence of bladder cancer in humans exposed to such compounds in the work place. The enzymatic activation involves first a $\mathrm{N}$-hydroxylation which is followed by a conjugation reaction, potentially leading to a good leaving group and the generation of a

$a, b, c)$<smiles>[X]C(C)=C(C)C</smiles>

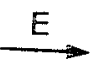

d)

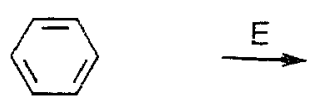

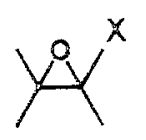

Fig. $2 a-d$. Part structures of carcinogens which can form an epoxide as reactive metabolite. a, b, c Activated olefins, d aromatic hydrocarbons; $X$ electrophilic substituent

Tabie 2. Group 2 of IARC 1980 list of carcinogens: substituted olefins (13 of 54)

Substitution

a) With chloride

b) With oxygen

c) Unsaturation

d) In aromatic system
18. Chlordane
47. Polychlorinated biphenyls
51. Trichloroethylene
54. Vinyl chloride
2. Aflatoxins
1. Acrylonitrite
19. Chloroprene
24. Diethylstilbestrol
50. Styrene
9. Benzene
44. Phenobarbitone
46. Phenytoin
49. Soots and tars 
nitronium ion (Fig. 3a). Upon delocalization of electrons, the positive charge can move into the aromatic system and the analysis of the reaction products with DNA indeed revealed adducts formed via exocyclic nitrogen and ortho-carbon. The IARC 1980 list of carcinogens contains as much as seven aromatic amines (Table 3). They are probably overrepresented with respect to their importance for the general population but they are so numerous in this list because industrial exposure to a carcinogen is much more easily detectable by an epidemiological analysis.

Alkyl hydrazines are not only experimental carcinogens but are found as drugs and dietary constituents, especially in mushrooms. A postulated enzymatic pathway involving both $\mathrm{N}$ - and C-hydroxylations can lead to the generation of alkyl diazohydroxide (Fig. 3b). The same type of proximate carcinogen is formed in the metabolic degradation of nitrosamines derived from secondary amines (Fig. 3c). For this reason these environmentally important carcinogens have been included in group 3 (Table 3 ).

a)<smiles>Nc1ccc2ccccc2c1</smiles><smiles>CC1C=c2ccccc2=CC1=N</smiles><smiles>ONc1ccc2ccccc2c1C(F)(F)F</smiles>

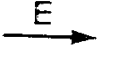<smiles>O=S(=O)(O)Nc1ccc2ccccc2c1</smiles>

b)
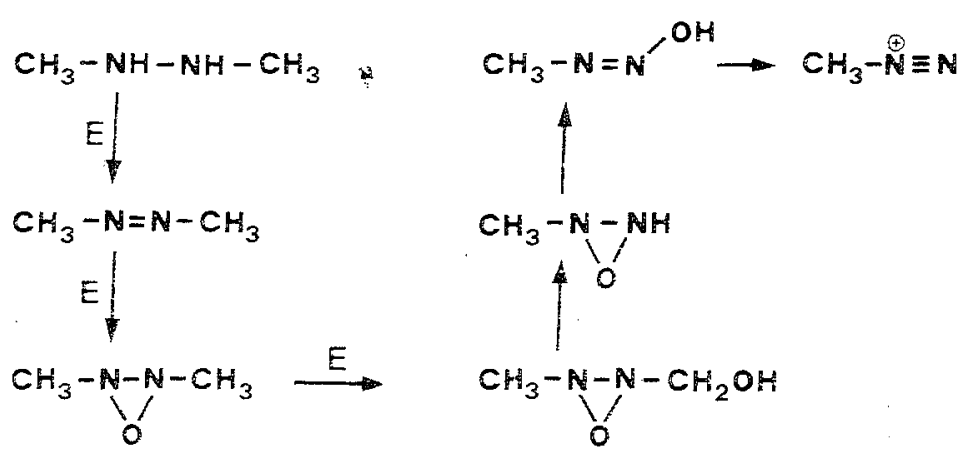

c)<smiles>CN(C)N=O</smiles><smiles>CN(CO)N=O</smiles>

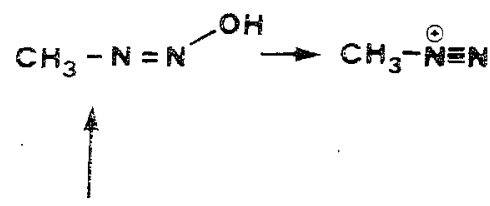

Fig. 3a-c. Metabolic activation of carcinogens via oxidation of nitrogen or of an alpha carbon to a nitrosated amine. a Beta-naphthylamine as an aromatic amine, $\mathbf{b} 1,2$-dimethylhydrazine; the epoxide stands also for the azoxy resonance structures, c dimethylnitrosamine 
Table 3. Group 3 of IARC 1980 list of carcinogens: aromatic amines, hydrazines, nitrosamines. Activation by oxidation of nitrogen or alpha-carbon to nitrogen ( 9 of 54)
a) Aromatic amines
3. 4-Aminobiphenyl
4. Amitrole
7./8. Auramine and its manufacture
10. Benzidine
39. 2-Naphthylamine
43. Phenacetin
45. N-Phenyl-2-naphthylamine
b) Hydrazines
33. Isoniazid
c) Nitrosamines
* Dimethylnitrosamine

Numbering according to IARC (1980)

* Additional carcinogen

\section{Group 4: Halogenated Hydrocarbons}

This is a highly complex class of carcinogens. Some can be dehydrohalogenated to an olefin (Fig. 4a; to a group 2 compound), some are known to undergo homolytic cleavage of a halogen-carbon bond and thus give rise to radicals able to react with critical target molecules or to lead to the generation of even more dangerous radicals (Fig. 4b). In addition to this potential formation of reactive intermediates, most of the halogenated hydrocarbons are well-known inducers of drug-metabolizing enzymes so that they have often been attributed, without experimental backup though, some type of co-carcinogenic activity. To make the situation even more complex, a number of these compounds have been shown to stimulate cell division and to act as tumor promoters in two-stage carcinogenicity tests (see Schulte-Hermann 1981). Some carcinogens of this class may therefore act on a variety of levels and it will be most challenging to determine the relative importance of the particular activities. However, one should not forget that the overall carcinogenic potency is low for this class of compounds and that many aspects may turn out to be species specific. It is therefore not surprising to see that the evidence for carcinogenicity in humans of the four members of this class, listed among the 54 (Table 4), has been termed inadequate by IARC (1980).

a)<smiles>ClC(Cl)=C(c1ccc(Cl)cc1)c1ccc(Cl)cc1</smiles>

b)

$\mathrm{CCl}_{4}$

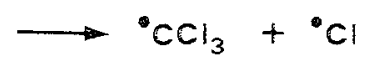

Fig. 4 a, b. Possible steps in the metabolic activation of halogenated hydrocarbons. aDDT dehydrohalogenated to an olefin, $b$ homolytic cleavage of carbon tetrachloride to radicals 
Table 4. Group 4 of IARC 1980 list of carcinogens: aliphatic halogenated compounds (4 of 54)
a) Dehydrohalogenation to olefin (group 2)
22. DDT
31. Hexachlorocyclohexane
b) Homolytic cleavage of $\mathrm{C}-\mathrm{Cl}$ bond to radical
15. Carbon tetrachloride
23. Dieldrin (also group 1)

Numbering according to IARC (1980)

\section{Group 5: Preceding Reactions Required}

This group of compounds requires some enzymatic modification of the molecule which is either not normally performed in the mammalian cell (nitro reduction or beta-glucosidase activity) or which does not center around the atom to become reactive (phosphoramide cleavage of a non-reactive mustard to liberate a reactive mustard or aromatization of a pyrrolizidine alkaloid to generate "benzylic" $-\mathrm{CH}^{+}-$and $\mathrm{CH}_{2}^{+}$). These reactions are depicted in Fig. 5 (a and c, b and d, respectively). Only two such examples have been found in the IARC 1980 list of carcinogens (Table 5, a and b) and two have been added for the sake of<smiles>O=[N+]([O-])c1ccccc1</smiles>

b)

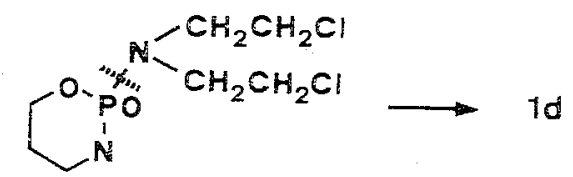

(3a)

\author{
a)
}

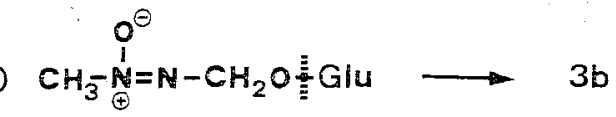

Fig. 5a-d. Selection of carcinogens known to require a preceding reaction not normally performed in the target cell (a, nitroreductase;

c, beta-glucosidase) or not at the atom to become electrophilic

(b, phosphoramide cleavage of cyclophosphamide to release a reactive mustard; $\mathrm{d}$, aromatization of pyrrolizidine alkaloid to generate benzyl analogues) 
completeness. Nitroreductase and beta-glucosidase both are activities present in microorganisms, and it cannot, therefore, be surprising that the genotoxicity of dinitrotoluene is no longer observed in germ-free animals (Mirsalis et al. 1982). It is also conceivable that the diet can have an indirect influence on the carcinogenicity of this type of compound by changing the number and composition of the gut microflora.

\section{Answer to Question 1: Sensitivity for Organic Compound is Very High}

Table 6 shows that it would be possible to identify as much as $72 \%$ of the 54 carcinogens of the IARC list and even $91 \%$ if only organic chemicals are considered. This sensitivity is even slightly better than with the Ames test if applied to this selection of carcinogens, because of the well-documented problems of the Ames test with halogenated compounds and hydrazines.(Rinkus and Legator 1979).

Table 6. Carcinogens of IARC 1980 list which could have been detected on the basis of structural elements

\begin{tabular}{lc}
\hline Group 1: Chemically reactive & 13 \\
Group 2: Substituted olefins & 13 \\
Group 3: Aromatic amines/hydrazines & 9 \\
Group 4: Aliphatic halogenated compounds & 3 new \\
Group 5: Nitroaromatics and miscellaneous & 1 new \\
Total & 39 of $54(72 \%)$ \\
If only organic carcinogens are taken & 39 of $43(91 \%)$ \\
\hline
\end{tabular}

The compounds of the IARC 1980 list which would not have been detected on the basis of structural elements as presented in groups $1-5$ are primarily inorganic and include derivatives of arsenic (no. 5 of the IARC list), beryllium (11), cadmium (14), chromium (20), lead (36), nickel (40 and nickel refining, $41)$, as well as asbestos (6) and hematite (29 and its mining, 30). One possible mechanism of carcinogenic action might involve an interaction with the DNA polymerase system and a resulting decrease of template fidelity but such activity is obviously not amenable to an analysis of electrophilicity. Only four organic chemical are missed; i.e., iron dextran (32), isopropyl oils and their manufacture (34 and 35), oxmetholone (42) and reserpine (48). The latter two are known to interfere with the endocrine system. They could therefore be carinogenic along the line of hormones and are detectable in chronic toxicity studies by their respective activities.

\section{Question 2: Jow Grood is the Specificity?}

The question of specificity deals with the problem of how many from a selection of non-carcinogens do not have structural elements placing them into one of 


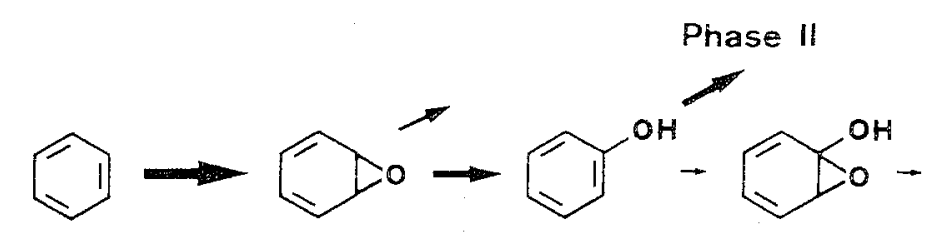

Fig. 6. Schematic metabolism of benzene, phenol, and toluene<smiles></smiles><smiles>O=C(O)c1ccccc1C=CC=[Pb]</smiles>

groups 1-5. A simple example shown in Fig. 6 illustrates the main problem. Benzene, toluene, and phenol would all have to be termed potential carcinogens, if the rules set out above are adopted and they are placed in group 2. Only benzene is a detectable systemic carcinogen (Dean 1978). This is mainly because the metabolism of the other two compounds goes primarily via true detoxication reactions, such as the oxidation of the side chain of toluene and conjugation reactions with phenol. The oxidation in the ring does occur with both but represents only a minor pathway.

One could think of introducing this type of knowledge into the evaluation and to state that alkylated or phenolic aromatic hydrocarbons are less dangerous than their parent compounds. This approach would not be of much quantitative value because of two main points.

Firstly, in some instances, such as with benz(a)anthracene derivatives, many methylated analogues are much more potent carcinogens (Oesch 1982), or, the conjugation reaction can even be a prerequisite for the formation of the ultimate reactive carcinogen, such as with the aromatic amines or with 1,2-dibromoethane, where the reaction with glutathione leads to a reactive brominated sulfur mustard.

Secondly, on a quantitative basis, all the modulations are not taken into account which ultimately determine the concentration of reactive metabolite at the target and the reactivity with DNA. The competing activation and inactivation processes are highly complex and controlled on many possible levels some of which are discussed in later presentations.

It therefore seems that only in vivo studies can possibly give a quantitative answer on the level of DNA damage exerted by reactive metabolites. The difference between benzene and toluene for instance becomes very clear when a DNA-binding assay is performed in rats. The binding to liver DNA was found to be at least 40 times higher for benzene (Lutz and Schlatter 1977) if compared with toluene (Table 7). 
Table 7. Covalent binding of $\left[{ }^{14} \mathrm{C}\right]$ benzene and $\left[\mathrm{p}^{3} \mathrm{H}\right]$ toluene to rat liver DNA after exposure in a closed inhalation system

\begin{tabular}{lccc}
\hline Compound & Benzene & Toluene \\
\hline Dose & 62 & 63 & 64 \\
$(\mathrm{mg} / \mathrm{kg})$ & 6.5 & 6.6 & 182 \\
$\left(10^{9} \mathrm{dpm} / \mathrm{kg}\right)$ & 9 & 11.5 & 5 \\
Period of exposure (h) & - & - & 0.6 \\
Formation of tritiated water (\% of dose) & & & \\
Liver DNA & 2.3 & 2.2 & 2.5 \\
In vial (mg) & 42 & 37 & 25 \\
Spec. act. (dpm/mg) & 1.7 & 1.5 & $<0.04$ \\
(CBI units) & & & \\
\hline
\end{tabular}

a Specific activity of DNA normalized by the dose administered and converted to molar units as defined in the list of abbreviations

\section{Answer to Question 2: Bad Specificity}

Therefore, it must be concluded that a large number of false positive answers will be generated if structure-activity considerations are used as a short-term test for carcinogenicity. Only in vivo experiments would accurately take account into the inactivation reactions which can be responsible for drastic reductions in the concentration of reactive metabolites at the target molecule. The specificity will therefore be bad, worse than with the Ames test where some inactivation processes, albeit distorted, are still taken into account. Because of the ready availability of the Ames test as a highly sensitive screening test for the production of metabolites damaging bacterial DNA, it would not seem that a sophisticated decision tree as set forth by Cramer et al. (1978) would be an alternative.

\section{Question 3: What is the Role of Reactive Metabolites in Over-all Carcinogenicity?}

Table 8 gives an outline of the possible modes of action of chemical carcinogens, divided into mutagenic and non-mutagenic events, each group being subdivided into possible specific activities. A compound exhibiting any one single activity could be found to increase the tumor incidence in a long-term bioassay on carcinogenicity if it is accepted that mutational events are to some extent unavoidable (Lutz 1982), and that the respective mutations can also form the basis for spontaneous tumor formation.

The reactive metabolites, discussed here, seem to act primarily by DNA binding. In addition, and as mentioned for the group of the halogenated hydrocarbons, there is the possibility of radical-induced damage also in other classes of compounds which might act via oxygen radical formation. Other papers in this volume will deal more thouroughly with this aspect. Besides these 
Table 8. List of possible modes of action of chemical carcinogens

\begin{tabular}{ll}
\hline Mutagenicity & $\begin{array}{l}\text { Non-mutagenicity } \\
\text { (modulation of mutational events) }\end{array}$ \\
\hline $\begin{array}{l}\text { Derived from } \\
\text { - DNA binding of test compound }\end{array}$ & At the level of \\
- Indirect DNA damage & Pre-mutagenicity \\
e.g., via oxygen radicals & more DNA binding, \\
Other mutagenic mechanisms & - Co-mutagenicity \\
& more rapid fixation of DNA damage \\
& (stimulation of cell division) \\
& - Post-mutagenicity \\
& better expression of critical \\
& mutation towards tumor growth \\
& (= promotion?) \\
\hline
\end{tabular}

two modes of obvious genotoxicity, a number of additional mechanisms on the chromatid and chromosome levels will lead to mutations in a broadest sense.

On the non-mutational side, modulations have to be dealt with on many levels. Enzyme induction is often referred to as a possible co-carcinogenic mechanism of action although all reports seem to show the opposite: a pretreatment of animals with enzyme inducers of any kind, so far, has always led to a reduction in the incidence of tumors induced by a subsequent dose of a DNA-binding carcinogen (Wattenberg 1978).

The step between DNA damage and mutation is dependent on the repair of the critical DNA damage, the mutagenicity of the various adducts, and on the rate of DNA synthesis, i.e., of the rate of cell division. Any influence which increases the cell division rate reduces the time available for repair and increases the probability for a fixation of the damage in the form of mutation. Hormones might therefore be found to be carcinogenic in an animal bioassay on the basis of their stimulatory effect on target cell division.

Some compounds listed among the 54 IARC carcinogens are known to lead to liver growth in rodents, such as hexachlorocyclohexane (HCH) or phenobarbital. Both have also been mentioned before because of their critical structural elements. It is therefore possible that these compounds act by more than one mode. The third question raised here will deal with the quantitative importance of a single activity like DNA binding.

$\mathrm{HCH}$ includes a group of isomers of which the gamma-isomer, called lindane, has useful pesticidal activities. The alpha-isomer is a moderately strong hepatocarcinogen, much stronger than the gamma-isomer for which the evidence for carcinogenicity is inconclusive. The tumor induction in mice is strongly dependent on the strain used. Both isomers could give rise to reactive metabolites via dehydrohalogenation to an olefin and epoxidation. A DNA binding assay in mouse liver was performed with oral administration of tritiated $\mathrm{HCH}$ and isolation of the DNA after $10 \mathrm{~h}$ (Sagelsdorff et al. 1983). The results support the view that DNA-binding cannot be the decisive mode of tumorigenic 
activity in that Covalent Binding Indices, CBI (see list of abbreviations for definition), of $\leqslant 0.26$ and $\leqslant 0.18$ were determined for the gamma- and the alpha-isomer, respectively. The alpha-isomer therefore did not give rise to a higher $\mathrm{CBI}$ although it is the much more potent carcinogen. Furthermore, it was shown that the CBI of gamma-HCH for mouse liver DNA was $\leqslant 0.28, \leqslant 0.26$, and $\leqslant 0.17$ in the NMRI, CF1, and B6C3F1 strain, i.e., similar DNA binding was found in all strains of mice tested although the CF1 mice are more susceptible than the others. In addition, the absolute level of DNA binding determined for the alpha-isomer is by a factor of about three orders of magnitude lower than with typical DNA-binding carcinogens of similar carcinogenic potency (Lutz 1982).

$\mathrm{HCH}$ isomers therefore seem not to belong to that population of carcinogens where DNA binding is the most important mode of action although DNA binding is possible from structure-activity considerations and measurable in vivo.

In conclusion, the carcinogenicity of a chemical must be considered to be the result of the sum of a variety of single contributing factors. The example given above with hexachlorocyclohexane is only one of a probably large number of cases where the reactive metabolites and the respective DNA binding cannot be the main mode of carcinogenic activity. Conversely, this means that the lack of structural elements known to become potentially reactive dose not guarantee the lack of a carcinogenic potential and it will be important to establish biological activities common to representatives of the non-reactive carcinogens.

\section{Answer to Question 3: Reactive Metabolites and Their DNA-Binding are not Always Decisive for a Carcinogenic Activity of a Chemical}

\section{References}

Ashby J (1978) Structural analysis as a means of predicting carcinogenic potential. Br J Cancer $37: 904-923$

Cramer GM, Ford RA, Hall RL (1978) Estimation of toxic hazard - a decision tree approach. Food Cosmet Toxicol 16:255-276

Dean BJ (1978) Genetic toxicology of benzene, toluene, xylenes and phenols. Mutat Res $47: 75-97$

IARC Working Group (1980) An evaluation of chemicals and industrial processes associated with cancer in humans based on human and animal data: IARC monographs volumes 1 to 20. Cancer Res $40: 1-12$

Lutz WK (1979) In vivo covalent binding of organic chemicals to DNA as a quantitative indicator in the process of chemical carcinogenesis. Mutat Res $65: 289-356$

Lutz WK (1982) Constitutive and carcinogen-derived DNA binding as a basis for the assessment of potency of chemical carcinogens. Adv Exp Med Biol 136B : 1349-1365

Lutz WK, Schlatter C (1977) Mechanism of the carcinogenic action of benzene: Irreversible binding to rat liver DNA. Chem Biol Interact 18:241-245

Miller JA (1970) Carcinogenesis by chemicals: an overview - G. H. A. Clowes memorial lecture. Cancer Res 30:559-576

Mirsalis JC, Hamm Jr TE, Sherill JM, Butterworth BE (1982) Role of gut flora in the genotoxicity of dinitrotoluene. Nature 295:322-323 
Oesch F (1982) Problems associated with the use of chemical class controls in absence of information on the underlying mechanism. In: Nicolini $C$ (ed) Chemical carcinogenesis. Plenum, New York, p 67

Rinkus SJ, Legator MS (1979) Chemical characterization of 465 known or suspected carcinogens and their correlation with mutagenic activity in the Salmonella typhimurium system. Cancer Res 39: 3289-3318

Sageldorff P, Lutz WK, Schlatter C (1983) The relevance of covalent binding to mouse liver DNA to the carcinogenic action of hexachlorocyclohexane isomers. Carcinogenesis 4:1267-1273

Schulte-Hermann R, Ohde G, Schuppler J, Timmermann-Trosiener I (1981) Enhanced proliferation of putative preneoplastic cells in rat liver following treatment with the tumor promoters phenobarbital, hexachlorocyclohexane, steroid compounds, and nafenopin. Cancer Res 41: 2556-2562

Wattenberg LW (1978) Inhibitors of chemical carcinogenesis. Adv Cancer Res 26: 197-226 